\title{
Renda do Petróleo: Uma revisão sistemática da literatura
}

Oil Rents: A systematic review of the literature

Renta del petróleo: Una revisión sistemática de la literatura

Recebido: 16/07/2021 | Revisado: 20/07/2021 | Aceito: 21/07/2021 | Publicado: 28/07/2021

Fernando Maciel

ORCID: https://orcid.org/0000-0002-6078-5183 Universidade Cândido Mendes, Brasil E-mail: maciel.frj@gmail.com

Marcelo Cruz

ORCID: https://orcid.org/0000-0001-5708-6552 Universidade Cândido Mendes, Brasil E-mail: mcruzflu@gmail.com

Vagner Vasconcelos

ORCID: https://orcid.org/0000-0003-4606-2816 Universidade Cândido Mendes, Brasil

E-mail: vagnervasconcelos@yahoo.com.br

Bruno Ferreira de Oliveira

ORCID: https://orcid.org/0000-0003-2727-774X Universidade Cândido Mendes, Brasil E-mail: brunooliver17@hotmail.com

Alberto Eduardo Besser Freitag ORCID: https://orcid.org/0000-0001-7182-1525 Universidade Cândido Mendes, Brasil E-mail: abesser@uol.com.br

Rodolfo Tomás da Fonseca Nicolay

ORCID: https://orcid.org/0000-0003-1325-5136 Universidade Cândido Mendes, Brasil E-mail: r-nicolay@hotmail.com

\begin{abstract}
Resumo
A renda do petróleo exerce impacto sobre os mecanismos de desenvolvimento dos países. Considerando a relevância do tema, o objetivo deste estudo é a realização de uma revisão da bibliografia sobre a renda do petróleo, sistematizando o conhecimento estabelecido e as agendas de pesquisa. A amostra utilizada consiste em 182 artigos coletados na Web of Science, no período de 1978 a agosto de 2020. Os resultados indicam o crescimento de artigos publicados a partir de 2014, período coincidente com a queda do preço do petróleo, que impactou os países cujo eixo dinâmico da economia reside neste setor. Dez países em termos de nacionalidade dos autores concentram 58,24\% dos autores dos artigos sobre o tema. O artigo com maior média de citação por ano é Regional Favoritism e Khalid Zaman foi identificado como o principal autor da amostra. As principais revistas científicas em termos de quantidade de artigos e de fator de impacto são, respectivamente, Resources Policy e Energy Economics. A rede de cocitação de autores indicou que os principais autores discutem as implicações relacionadas com a abundância dos recursos naturais. A rede de co-ocorrência de palavras-chave com a indicação de incidência em períodos recentes, evidenciou a direção dos estudos para temas relacionados aos impactos sobre o crescimento, a utilização de métodos empíricos para os estudos sobre o tema, discussão sobre a abundância, dependência e maldição dos recursos naturais, financiamento do desenvolvimento, relação entre capital humano e renda do petróleo, bem como temas ligados ao meio ambiente e sustentabilidade.
\end{abstract}

Palavras-chave: Renda do petróleo; Bibliometria; Redes; Abundância dos recursos naturais.

\section{Abstract}

Oil rents has an impact on the development mechanisms of countries. Considering the relevance of the topic, the objective of this study is to conduct a review of the literature on oil revenue, systematizing the established knowledge and research agendas. The sample used consists of 182 articles collected from Web of Science, in the period from 1978 to August 2020. The results indicate the growth of articles published from 2014, a period coinciding with the fall in oil prices, which impacted countries whose dynamic axis of the economy resides in this sector. Ten countries in terms of authors' nationality concentrate $58.24 \%$ of the authors of the articles on the topic. The article with the highest average citation per year is Regional Favoritism and Khalid Zaman was identified as the main author of the sample. The top scientific journals in terms of quantity of articles and impact factor are Resources Policy and Energy Economics, respectively. The author cocitation network indicated that the leading authors discuss the implications related to natural resource abundance. The co-occurrence network of keywords with the indication of incidence in 
recent periods, evidenced the direction of studies towards themes related to impacts on growth, the use of empirical methods for studies on the subject, discussion on the abundance, dependence and curse of natural resources, financing of development, the relationship between human capital and oil income, as well as themes related to the environment and sustainability.

Keywords: Oil rents; Bibliometrics; Networks; Natural resource abundance.

\section{Resumen}

La renta del petróleo repercute en los mecanismos de desarrollo de los países. Teniendo en cuenta la relevancia del tema, el objetivo de este estudio es realizar una revisión de la bibliografía sobre la renta petrolera, sistematizando los conocimientos establecidos y las agendas de investigación. La muestra utilizada consta de 182 artículos recogidos de Web of Science, en el período comprendido entre 1978 y agosto de 2020. Los resultados indican el crecimiento de los artículos publicados a partir de 2014, periodo que coincide con la caída de los precios del petróleo, que impactó en los países cuyo eje dinamizador de la economía reside en este sector. Diez países en cuanto a la nacionalidad de los autores concentran el 58,24\% de los autores de los artículos sobre el tema. El artículo con la mayor media de citas por año es Favoritismo Regional y Khalid Zaman fue identificado como el autor principal de la muestra. Las principales revistas científicas en cuanto a cantidad de artículos y factor de impacto son Resources Policy y Ernegy Economics, respectivamente. La red de co-ocurrencia de autores indicó que los autores principales discuten las implicaciones relacionadas con la abundancia de recursos naturales. La red de co-ocurrencia de palabras clave con la indicación de incidencia en los últimos períodos, evidenció la orientación de los estudios hacia temas relacionados con los impactos en el crecimiento, el uso de métodos empíricos para los estudios sobre el tema, la discusión sobre la abundancia, dependencia y maldición de los recursos naturales, la financiación del desarrollo, la relación entre el capital humano y la renta petrolera, así como los temas vinculados al medio ambiente y la sustentabilidad.

Palabras clave: Renta del petróleo; Bibliometría; Redes; Abundancia de recursos raturales.

\section{Introdução}

O petróleo é uma mistura complexa de hidrocarbonetos que precisam ser separados por diversos processos para formar os derivados utilizados pelos consumidores e pela indústria em geral (ANP, 2020). Sendo uma commodity de alto valor dentre os recursos naturais, a exploração de petróleo é uma atividade que resulta em níveis elevados de recursos financeiros para os países produtores. Dada sua influência nas economias globais, a análise dos impactos causados por esta renda tem sido objeto frequente de estudos sob os mais diversos aspectos.

A indústria de Petróleo e Gás é uma atividade produtiva que integra diversas áreas de conhecimento e mobiliza expressivos recursos humanos e econômicos que sustentam todas as operações ao longo de sua extensa cadeia de valor. São realizadas atividades de Upstream - compreendidas pela exploração, desenvolvimento, produção e transporte para beneficiamento; e Downstream - transformação do petróleo em produtos para uso específico e distribuição ao consumidor final. A atuação coordenada de diversos atores é um fator crítico para o sucesso do setor de Petróleo e Gás, dado que o domínio destas tecnologias podem ser cruciais para o desenvolvimento de algumas economias.

Segundo o World Energy Outlook 2020 da International Energy Agency (IEA, 2020), estima-se que serão investidos aproximadamente US\$ 17 trilhões na indústria de petróleo e gás no horizonte de 2020 a 2040, no qual do montante indicado, US\$1,5 trilhões estão destinados à Américas do Sul e Central. No Brasil, a indústria de petróleo e gás tem sido responsável por grande parte dos investimentos diretos realizados no país. Segundo o Observatório do Setor de Petróleo e Gás do Instituto Brasileiro de Petróleo (IBP), o setor atraiu mais de US\$ 48 bilhões no período ao longo da última década. Estima-se ainda que o setor seja responsável por 53\% de todo o investimento a ser realizado na indústria do país no triênio 2019-2022 (IBP, 2020).

A aplicação destes recursos financeiros impacta significativamente a economia do país ao estimular a demanda de máquinas, equipamentos, insumos e outras cadeias produtivas. Para os entes públicos, a indústria de petróleo e gás é uma importante geradora de tributos e de empregos formais, que em meados de 2019 totalizavam 38,7 milhões de postos de trabalho no Brasil. Adicionalmente, ressalta-se a relevância do petróleo e seus derivados no resultado das exportações, posicionando-se em $2^{\circ}$ lugar no ranking de participação das exportações em 2018 (IBP, 2020). 
De forma preliminar, é importante observar que a distribuição destes recursos ao longo do globo terrestre não ocorre de maneira uniforme. Os países que concentram as maiores reservas de petróleo - considerando o volume de reservas provadas - são Venezuela, Arábia Saudita, Canadá, Irã e Iraque, porém há ainda volumes expressivos de petróleo em países da África, Ásia e Europa (BP, 2020). Esta restrição de seu estoque na natureza e a consequente indisponibilidade do recurso para as gerações futuras pela sua extração no período presente, ensejam uma compensação financeira (renda) ao proprietário da jazida pela sua redução ao longo do tempo (Postali, 2011). Diante desta decisão de utilização de valer-se do recurso no presente tornando-o indisponível para as próximas gerações, a alocação destas rendas deve ser transparente, clara e com amplo controle social e institucional.

Neste sentido, o impacto causado pelas rendas advindas de recursos naturais tem sido objeto de diversos estudos realizados pela comunidade científica desde a década de 1980, seja na perspectiva sobre os impactos sobre os mecanismos de crescimento e desenvolvimento dos países, dado que o fluxo destas rendas pode impulsionar padrões de investimento e consumo por meio de financiamento público e privado mais elevados (Sachs, 2007), sobre os impactos nos aspectos institucionais (Acemoglu, Johnson \& Robinson, 2001), seja pela sua influência sobre os sistemas políticos dos países (Aytaç, 2016; Okada, 2017), além de relacionar as consequências sobre o meio ambiente (Ilhan, 2017).

Assim, o objetivo deste artigo é realizar uma análise bibliométrica dos estudos realizados sobre rendas do petróleo de forma a classificar as principais áreas de interesse e suas diversas correlações, de forma a identificar o conhecimento estabelecido sobre o tema e a direção que os estudos tem adotado em períodos recentes, para identificar a perspectiva de direcionamento das agendas de pesquisa. Além disso, o presente estudo é útil para formuladores de políticas públicas, no sentido de apresentar a base intelectual e analítica sobre a renda do petróleo, de forma influenciar positivamente na qualidade das políticas públicas versadas sobre o presente tema.

A amostra utilizada no presente estudo consiste em 182 artigos coletados da base de dados Web of Science (WoS), no período de 1978 a agosto de 2020, utilizando métricas bibliométricas relacionados com os artigos científicos publicados, sobre as referências intelectuais e analíticas destes artigos, bem como as palavras-chave dos respectivos artigos. além desta seção introdutória, o artigo apresenta uma breve definição sobre renda do petróleo, seguido de uma seção metodológica, análise e discussão dos resultados e das considerações finais.

Além desta seção introdutória, a estrutura deste trabalho aborda na segunda seção a definição de renda do petróleo, reforçando os seus aspectos conceituais. A terceira sessão destaca as bases de dados utilizadas, bem como os aspectos metodológicos utilizadas para a análise bibliográfica do estudo. A quarta sessão analisa os fatores bibliométricos, a partir das redes de citações e palavras-chave. Por fim, as conclusões e referências.

\section{Definição de Renda do Petróleo}

A disponibilidade de recursos naturais poderia ser compreendida como um fator de "sorte" para o desenvolvimento econômico de um dado país. Eles podem ser classificados por: (i) sua reprodutibilidade - renovável ou não renovável; (ii) por tipo - mineral, agrícola, pecuário ou silvicultura; e (iii) por uso energético - combustíveis fósseis (petróleo, gás e carvão) ou minerais não energéticos (por exemplo cobre, níquel, entre outros) (Reis, 2012). Por não ser fruto de um processo produtivo, sob a ótica da economia pode ser tratada como "ativo" ou um diferente tipo de riqueza capaz de produzir impactos no desenvolvimento econômico (Humphreys et al., 2007). Porém, é preciso considerar a ideia de que recursos naturais não são uma "dádiva" da natureza, mas antes uma riqueza que só se efetiva com capital, tecnologia e conhecimento (Pamplona \& Cacciamali, 2017).

Há algumas peculiaridades na exploração de recursos naturais que tem levado a diversos estudos sobre o tema. Postali (2011) considera que a peculiaridade fundamental deste tipo de atividade é a limitação física de seu estoque na natureza e a 
consequente indisponibilidade do recurso para as gerações futuras caso ele seja extraído no presente. Assim, haveria uma compensação ao proprietário da jazida pela redução no seu valor devido à extração de seus recursos - denominada renda.

Uma das primeiras teorias sobre este conceito, preconizada por Hotelling (1931), propunha estabelecer um manejo ótimo de recursos escassos e tenta determinar as condições para uma depleção ótima no tempo do recurso. Já a proposição de que tal renda seja investida em desenvolvimento e venha garantir o bem-estar social tornou-se conhecida como a Regra de Hartwick (1977), no qual garante que o consumo entre gerações, na ausência de crescimento populacional e progresso tecnológico, seja constante ao longo do tempo. Logo, seria necessário transformar as riquezas naturais em insumo produtivo para que as gerações futuras não sejam penalizadas pelo consumo do recurso esgotável no presente.

Para Cruz (2009), uma das principais alternativas a essa gestão sustentável é a transformação de recursos finitos em insumos permanentes que possam gerar fluxos de renda constantes para as gerações futuras. No caso brasileiro, a Constituição Federal de 1988 estabelece em seu Artigo 20 "os recursos naturais da plataforma continental e da zona econômica exclusiva" são bens da União e assegura participação aos Estados, ao Distrito Federal e aos Municípios, bem como a órgãos da administração direta da União no resultado da exploração de petróleo ou gás natural, de recursos hídricos para fins de geração de energia elétrica e de outros recursos minerais.

Neste sentido, seguem abaixo algumas das rendas e suas definições, incidentes nas operações relacionadas a atividade petrolífera:

a. Compensação Financeira pela Exploração de Recursos Minerais (CFEM): trata-se de contrapartida pela exploração econômica de recursos em minas, jazidas, salinas e outros depósitos minerais do território nacional;

b. Bônus de Assinatura: valor pago pela concessionária vencedora de processo licitatório visando a permissão para realizar atividades de pesquisa e exploração em determinada área (terrestre ou marítima);

c. Pagamento pela ocupação ou retenção de área - royalties: são uma quantia paga por alguém a um proprietário pelo direito de uso, exploração e comercialização de um bem; e

d. Participação especial: tributo cobrado das concessionárias sobre o campo que apresentar grandes volumes de produção ou rentabilidade.

\section{Metodologia}

Os estudos bibliométricos utilizam uma diversidade de métricas e indicadores ligados à mensuração da atividade científica, com o objetivo de apresentar uma análise sistematizada da bibliografia associada à área de pesquisa objeto de estudo, de forma a identificar a organização teórica e seu respectivo desenvolvimento temporal, bem como evidenciar hiatos e nichos de oportunidades para o desenvolvimento futuro de agendas de pesquisa (Ruíz-Navarro, 2004; Do Prado et al, 2016).

Segundo Chain et al. (2019), as análises de cocitações, coautorias, palavras-chave e das revistas científicas consistem nos principais indicadores bibliométricos para a revisão sistemática da bibliografia especializada. De forma complementar, a utilização da Análise de Redes Sociais (ARS) nos estudos bibliométricos se revelam úteis para apresentar de forma visual, padrões de conexões entre os agentes e de desenvolvimento científico (Van Eck, 2014; Barbosa; Reinert 2014).

A revisão sistemática da bibliografia relacionada com a temática da renda do petróleo foi realizada na base de dados da Web of Science (WoS), sem restrição temporal, para identificação do início dos estudos sobre o tema e sua evolução. Além disso, as informações descritivas dos principais artigos científicos e revistas também são baseados pelas informações de busca apresentados pela WoS. Cabe salientar que a utilização da base de dados da WoS se deve a sua completude e credibilidade, contemplando dados disponíveis em outras bases como por exemplo a Scopus (Hassan; Haddawy; Zhu, 2014). 
Cabe salientar que a busca dos artigos foi realizada até dia 04 de agosto de 2020, cujo termo de busca utilizado foi 'Oil rents', 'Natural Resource Rents', sendo estes os termos representativos para a discussão da renda do petróleo e seus impactos sobre a sociedade (Sachs, 2007). Desta forma, foram identificados 182 artigos na base da WoS, de acordo com o protocolo de busca apresentado no Quadro 1 abaixo.

Quadro 1. Protocolo de busca adotada para análise bibliométrica.

\begin{tabular}{|c|c|}
\hline ETAPAS & DESCRIÇÃO DAS ETAPAS \\
\hline $\begin{array}{l}1^{\text {a }} \text { Etapa - Início do levantamento } \\
\text { bibliográfico }\end{array}$ & $\begin{array}{l}\text { - Pesquisa por palavra-chave: } \\
\text { i) 'Oil rents'; e } \\
\text { ii) 'Natural Resource Rents' foram utilizadas no mecanismo de busca } \\
\text { estão relacionadas com a discussão do tema. } \\
\text { - Protocolo de busca na WoS: } \\
\text { TS=(Oil rents OR Natural Resource Rents) } \\
\text { Refined by: DOCUMENT TYPES=( ARTICLE ) } \\
\text { Timespan=All years. } \\
\text { Indexes=SCI-EXPANDED, SSCI, A\&HCI, CPCI-S, CPCI-SSH, ESCI. }\end{array}$ \\
\hline $\begin{array}{l}2^{\mathrm{a}} \text { Etapa - Pesquisa } \\
\text { encontrados }\end{array}$ & - Lista dos artigos para revisão de literatura: 182 artigos \\
\hline $3^{\text {a }}$ Etapa - Análise Bibliométrica & $\begin{array}{l}\text { - Informações Bibliométricas: } 182 \text { artigos. Os artigos encontrados foram } \\
\text { devidamente analisados e todos os trabalhos refletem a discussão do tema } \\
\text { da renda do petróleo. }\end{array}$ \\
\hline $4^{\text {a }}$ Etapa - Seleção de documentos & Documentos para construção analítica - 182 artigos \\
\hline $5^{\text {a }}$ Etapa - Análise documental & Informações baseadas na base analítica \\
\hline
\end{tabular}

Fonte: Autores.

Quanto aos indicadores bibliométricos, o presente artigo se baseará em suas análises nos seguintes indicadores: i) evolução temporal da quantidade de artigos publicados sobre a renda do petróleo na WoS; ii) identificação da nacionalidade dos autores dos artigos relacionados com a renda do petróleo; iii) principais artigos da amostra em termos de citações média por ano; iv) principais autores em termos de quantidade de artigos da amostra; e v) principais periódicos científicos associados aos artigos da amostra (Chain et al., 2019).

Para a análise de redes aplicado ao presente estudo bibliométrico, serão utilizados os dados de cocitação dos autores das referências dos artigos, de forma a identificar as principais bases intelectuais para o desenvolvimento dos estudos associados à renda do petróleo, bem como a rede de co-ocorrência de palavras-chave, apresentando ao mesmo tempo, as principais palavras-chave em termos de ocorrência e de incidência ao longo do tempo (Van Eck; Waltman, 2010; Van Eck., 2014). O software utilizado para a criação de redes com os artigos encontrados na base de dados da WoS é o VOSviewer (https://www.vosviewer.com/), programa este bastante utilizado para construção de redes e análise bibliométrica (Van Eck; Waltman, 2010; Van Eck et al., 2010).

\section{Resultados e Discussão}

\subsection{Análise dos Indicadores Bibliométricos}

Na perspectiva geral, o primeiro ano no qual foi encontrado nos periódicos indexados a WoS relacionado com a renda do petróleo foi em 1978, onde até os anos 2000, o referido tema foi pouco pesquisado. De acordo com o Gráfico 1 abaixo, observa-se que boa parte das publicações relacionadas com o tema estão concentradas em períodos recentes, onde 74,18\% dos artigos foram publicados a partir do ano de 2014. 
Gráfico 1. Evolução do Número de publicações sobre Renda do Petróleo da base de dados da WoS.

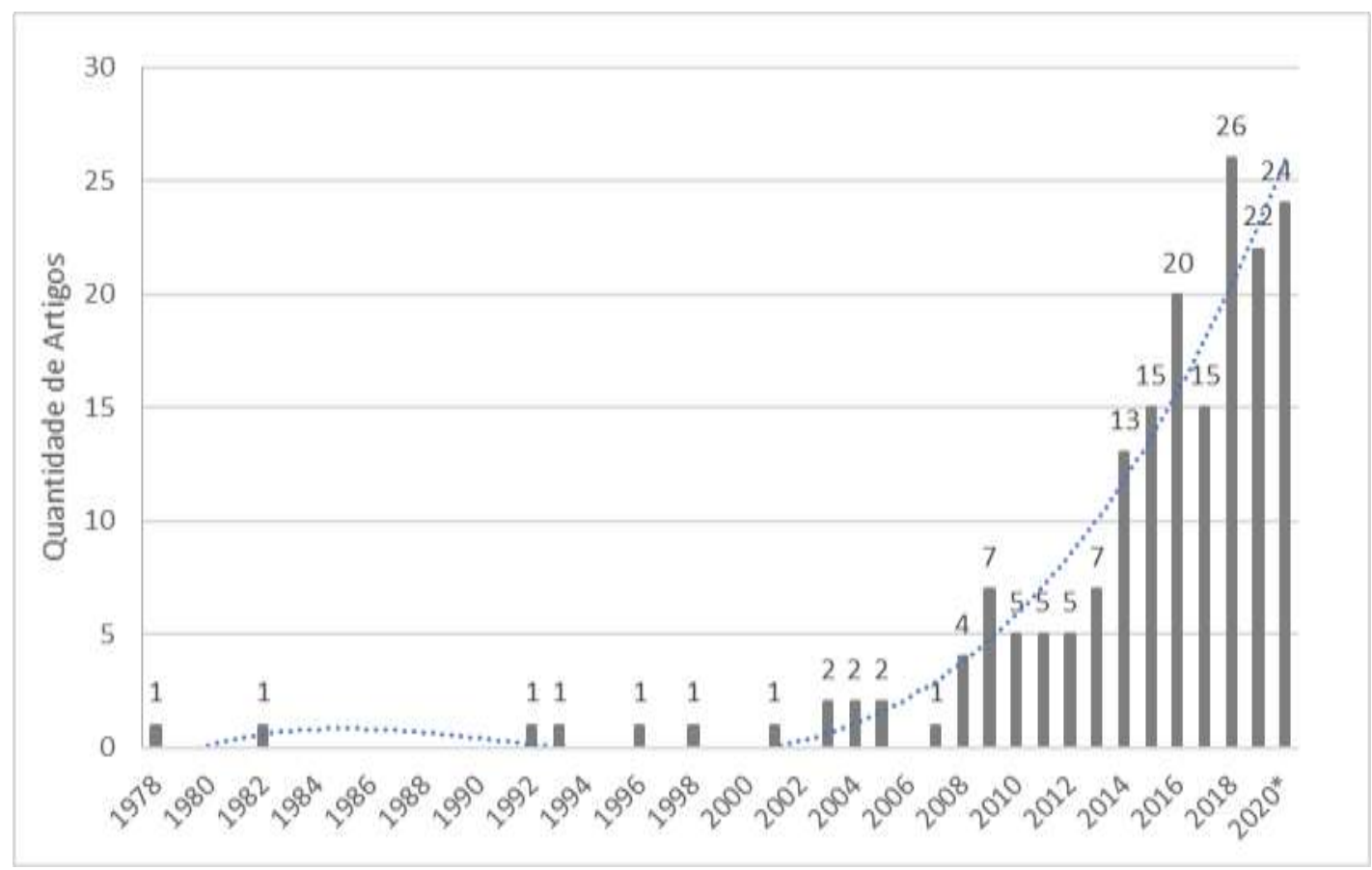

(*) Nota: a quantidade de artigos do ano de 2020 é o acumulado até a data do levantamento na WoS, realizado em 04 de agosto de 2020. Fonte: Autores.

Um ponto interessante sobre o crescimento elevado de publicações a partir do período de 2014 é que neste período, foi verificado um impacto importante da redução sistemática dos preços das commodities, sobretudo o preço dos barris de petróleo. O colapso de preços no final de 2014 é uma consequência imediata do profundo desequilíbrio entre oferta e demanda global de petróleo em que um grande excesso de capacidade de produção contrasta com uma desaceleração acentuada da demanda. Acrescente-se a isso a recusa da Arábia Saudita e outros produtores da OPEP em continuar exercendo seu papel tradicional de regulador do balanceamento entre oferta e demanda de petróleo por meio de cortes na produção (Pedrosa; Corrêa, 2016), que afetaram significativamente a estrutura de custos e estratégias de desenvolvimento de países produtores de petróleo, afetando o comportamento da aferição das rendas do petróleo para as regiões produtoras.

$\mathrm{Na}$ amostra referente aos artigos relacionados com o tema da renda do petróleo, foram encontrados 273 autores para os 182 artigos selecionados na base de dados da WoS, onde temos uma razão de 1,50 autores por artigo. Em relação à nacionalidade dos autores, verifica-se que aproximadamente $30 \%$ dos autores são dos EUA e Inglaterra, conforme apresentado pela Tabela 1 . 
Tabela 1. Distribuição dos autores por nacionalidade referente aos artigos sobre renda do petróleo da Base de dados da WoS e Recurso natural predominante em cada país.

\begin{tabular}{lcc}
\hline \multicolumn{1}{c}{ Países } & $\begin{array}{c}\text { Qtde. de } \\
\text { Autores }\end{array}$ & $\begin{array}{c}\text { \% de part. dos } \\
\text { autores }\end{array}$ \\
\hline EUA & 46 & $16,85 \%$ \\
Inglaterra & 35 & $12,82 \%$ \\
Alemanha & 16 & $5,86 \%$ \\
Paquistão & 12 & $4,40 \%$ \\
China & 9 & $3,30 \%$ \\
Turquia & 9 & $3,30 \%$ \\
Canadá & 8 & $2,93 \%$ \\
Nigéria & 8 & $2,93 \%$ \\
Arábia Saudita & 8 & $2,93 \%$ \\
África do Sul & 8 & $2,93 \%$ \\
\hline Total Top 10 & $\mathbf{1 5 9}$ & $\mathbf{5 8 , 2 4 \%}$ \\
\hline Total Outros Países (47) & 114 & $41,76 \%$ \\
\hline Total Geral & $\mathbf{2 7 3}$ & $\mathbf{1 0 0 , 0 0 \%}$ \\
\hline
\end{tabular}

Fonte: Autores, a partir da WoS.

Além disso, foi identificado entre os principais países em termos de nacionalidade dos autores a Alemanha, Paquistão, China, Turquia, Canadá, Nigéria, Arábia Saudita e África do sul, que em conjunto com os Estados Unidos e a Inglaterra, concentram 58,24\% dos autores relacionados com os artigos sobre renda do petróleo, países estes que possuem uma produção relevante de petróleo e gás natural.

Outro ponto a destacar é a forte presença de autores oriundos de países em desenvolvimento, onde dentre os principais países apresentados, sete são classificados como países em desenvolvimento. Este elevado interesse pode ser influenciado pelas estratégias de crescimento e desenvolvimento econômico pautados nos recursos naturais, cujo eixo dinâmico da economia está centrada neste segmento produtivo, caracterizando vantagens comparativas relevantes no comércio internacional.

Em relação aos artigos que possuem os maiores indicadores de citações média por ano dentre a amostra de artigos relacionados com a renda do petróleo, observa-se que a amplitude temporal de publicações destes artigos está entre os anos de 2008 e 2020. Os temas apresentados pelos artigos constantes na Tabela 2.

Para Hodler e Raschky (2014), a maior média de citações por ano (20), indica que alguns líderes políticos escolhem políticas que beneficiam principalmente suas regiões preferidas, sendo denominado favoritismo regional. A análise foi baseada em um conjunto de dados em painel com 38.427 regiões subnacionais em 126 países e observações anuais de 1992 a 2009 . Os autores demonstraram que a dinâmica do favoritismo regional é tal que os líderes políticos precisam de alguns anos antes de se envolverem com sucesso no favoritismo regional e, então tornarem-se cada vez melhores no apoio à sua região. Os resultados corroboram para a sustentabilidade política nas regiões analisadas.

Os autores fornecem algumas evidências de que a ajuda externa e as rendas do petróleo tendem a alimentar a busca de renda e o favoritismo regional em países pouco institucionalizados, mas não em países com instituições políticas comparativamente melhores. As informações sobre os principais artigos em termos de citações média por ano estão apresentadas na Tabela 2. 
Tabela 2. Artigos com maior citação média por ano da base de dados da WoS.

\begin{tabular}{|c|c|c|c|c|}
\hline Artigo & Autores & Revista Científica & $\begin{array}{c}\text { Média } \\
\text { Citações por } \\
\text { Ano } \\
\end{array}$ & Citações \\
\hline Regional Favoritism & $\begin{array}{l}\text { Hodler \& Raschky } \\
\qquad(2014)\end{array}$ & $\begin{array}{l}\text { Quarterly Journal of } \\
\text { Economics }\end{array}$ & 20,00 & 140 \\
\hline The curse of aid & $\begin{array}{l}\text { Djankov, Montalvo } \\
\text { \& Reynal-Querol } \\
\text { (2008) }\end{array}$ & $\begin{array}{l}\text { Journal of Economic } \\
\text { Growth }\end{array}$ & 17,46 & 227 \\
\hline $\begin{array}{l}\text { Leader Survival, Revolutions, and the } \\
\text { Nature of Government Finance }\end{array}$ & $\begin{array}{l}\text { De Mesquita \& } \\
\text { Smith (2010) }\end{array}$ & $\begin{array}{l}\text { American Journal of } \\
\text { Political Science }\end{array}$ & 13,18 & 145 \\
\hline $\begin{array}{l}\text { An empirical note on comparison between } \\
\text { resource abundance and resource } \\
\text { dependence in resource abundant countries }\end{array}$ & $\begin{array}{l}\text { Shahbaz et al. } \\
\qquad(2019)\end{array}$ & Resources Policy & 11,50 & 23 \\
\hline $\begin{array}{l}\text { Of rulers, rebels, and revenue: State } \\
\text { capacity, civil war onset, and primary } \\
\text { commodities }\end{array}$ & Thies (2010) & $\begin{array}{l}\text { Journal of Peace } \\
\text { Research }\end{array}$ & 9,09 & 100 \\
\hline $\begin{array}{l}\text { Modeling the nexus between coal } \\
\text { consumption, FDI inflow and economic } \\
\text { expansion: does industrialization matter in } \\
\text { South Africa? }\end{array}$ & $\begin{array}{l}\text { Udi, Bekun \& } \\
\text { Adedoyin (2020) }\end{array}$ & $\begin{array}{l}\text { Environmental Science } \\
\text { and Pollution Research }\end{array}$ & 9,00 & 9 \\
\hline $\begin{array}{l}\text { The Big Oil Change A Closer Look at the } \\
\text { Haber-Menaldo Analysis }\end{array}$ & $\begin{array}{l}\text { Andersen \& Ross } \\
\quad(2014)\end{array}$ & $\begin{array}{l}\text { Comparative Political } \\
\text { Studies }\end{array}$ & 8,86 & 62 \\
\hline $\begin{array}{l}\text { Testing the neocon agenda: Democracy in } \\
\text { resource-rich societies }\end{array}$ & $\begin{array}{l}\text { Collier \& Hoeffler } \\
\qquad(2009)\end{array}$ & $\begin{array}{l}\text { European Economic } \\
\text { Review }\end{array}$ & 8,25 & 99 \\
\hline $\begin{array}{l}\text { Triangular relationship among energy } \\
\text { consumption, air pollution and water } \\
\text { resources in Pakistan }\end{array}$ & Khan et al. (2016) & $\begin{array}{l}\text { Journal of Cleaner } \\
\quad \text { Production }\end{array}$ & 8,20 & 41 \\
\hline $\begin{array}{l}\text { Political Survival and Endogenous } \\
\text { Institutional Change }\end{array}$ & $\begin{array}{l}\text { De Mesquita \& } \\
\text { Smith (2008) }\end{array}$ & $\begin{array}{l}\text { Comparative Political } \\
\text { Studies }\end{array}$ & 8,17 & 98 \\
\hline
\end{tabular}

Fonte: Autores.

Com 17,46 citações médias por ano, Djankov, Montalvo e Reynal-Querol (2008) investigam a relação entre a ajuda externa e instituições políticas, considerando que este recurso estrangeiro “inesperado" pode resultar no mesmo comportamento verificado na bibliografia relacionada com a "maldição dos recursos naturais". Utilizando dados de painel para 108 países no período de 1960 a 1999, as descobertas dos autores sugerem que a ajuda externa prejudica as instituições democráticas. Os resultados demonstram que se a participação média da ajuda externa sobre o PIB de um país fosse de 1,9\% no período de 196-1999, o país receptor teria passado do nível médio de democracia nos países beneficiados no ano inicial para uma ausência total de instituições democráticas. Já o efeito do petróleo no longo prazo é menos importante, se o valor médio das receitas do petróleo sobre o PIB for de 12,2\% ao longo do período, o país destinatário passará do nível médio de democracia nos países destinatários no ano inicial para uma ausência total de democracia.

De Mesquita e Smith (2010), com 13,18 citações médias por ano, examinam como as instituições políticas e a estrutura das finanças do governo permitem que os líderes políticos enfrentem vários riscos de deposição. Os autores expõem que os líderes podem atenuar as ameaças revolucionárias aumentando a provisão de bens públicos, de forma que os cidadãos fiquem saciados, ou suprindo sua capacidade de organização. Os testes de sobrevivência do líder indicam que as ameaças 
revolucionárias aumentam a probabilidade de deposição de líderes não democráticos. Os líderes com acesso a recursos como ajuda estrangeira ou rendas de recursos naturais (como, por exemplo, o petróleo) estão mais bem equipados para sobreviver a essas ameaças e evitar a ocorrência dessas ameaças (De Mesquita \& Smith, 2010).

Com 11,50 citações médias por ano, Shahbaz et al. (2019) discutem a relação entre abundância de recursos e dependência de recursos em países com abundância de recursos no crescimento econômico, utilizando dados em painel para 35 países no período de 1980-2015. Destaca-se que existe uma presença transversal entre os países, confirmando que um choque em um dos países selecionados pode facilmente se espalhar para outros e na hipótese da maldição dos recursos, os autores descobrem que a abundância dos recursos naturais contribui para o crescimento econômico, mas a dependência dos recursos naturais, impede as atividades econômicas, e, portanto, retarda o crescimento.

Thies (2010), com média anual de 9,09 citações, investiga a relação entre o início da guerra civil e a capacidade do Estado, incluindo o papel desempenhado pelos produtos primários nessa relação. Os produtos primários são uma fonte de receita natural para os governantes e rebeldes, portanto, são um ponto focal útil para estudar a relação entre a capacidade do Estado e o início da guerra civil. Muitas das commodities primárias testadas nesses modelos impactam positivamente a capacidade do Estado em termos fiscais.

Com 9,00 citações médias por ano, Udi, Bekun e Adedoyin (2020), estudam o papel da industrialização no nexo energia-crescimento-investimento direto externo (IDE) para África do Sul, no período de 1970 a 2018 e investigam a relação de longo prazo ente as séries. Os resultados confirmaram que a industrialização não é um fator determinante para atrair a entrada de IDE para a África do Sul e como segunda implicação é que melhorar as condições das indústrias locais, não é uma pré-condição para atrair novos investidores potenciais e nem desenvolve a capacidade de absorção da economia. Ademais, um aumento na renda total dos recursos naturais afeta positivamente o PIB no curto e longo prazo.

Com 8,86 citações médias por ano, Andersen \& Ross (2014) estudou a relação da riqueza do petróleo e a tendência ao bloqueio nas transições democráticas, usando dados históricos desde 1800 e concluíram que não há "maldição de recursos". A partir da revisão destes dados e modelos e sua assertividade para o período antes da década de 1970, mas desde cerca de 1980, houve uma maldição de recursos pronunciada. Os autores argumentam que a riqueza do petróleo só se tornou um empecilho para transições democráticas após os eventos transformadores da década de 1970, que permitiram aos governos dos países em desenvolvimento capturar as rendas do petróleo que antes eram desviados por empresas estrangeiras (Andersen \& Ross, 2014).

Collier e Hoeffler (2009), com média de 8,25 citações, estudam se o efeito da democracia no desempenho econômico é distinto em sociedades com abundância de recursos naturais. Com dados para países entre o período de 1970 a 2001. Os resultados indicam que nos países em desenvolvimento, a combinação de altas rendas de recursos naturais e sistemas democráticos abertos tem reduzido o crescimento (Collier \& Hoeffler, 2009).

Com base nas variáveis críticas da literatura, Khan et al. (2016), com 8,20 citações médias por ano, estudou a relação causal entre consumo de energia, poluição do ar, recursos hídricos e renda dos recursos naturais no Paquistão. O trabalho analisou a relação de longo prazo abrangendo um período de 38 anos, de 1975 a 2012. Os resultados mostram que o consumo de energia e os recursos hídricos têm relação positiva significativa com a poluição do ar, tanto a curto prazo quanto a longo prazo.

Por fim, com média de 8,17 citações, Mesquita e Smith (2008) testaram empiricamente como as condições políticas e econômicas em termos de fornecimento de bens públicos essenciais, apresentaram dependência econômica de fontes gratuitas como petróleo e ajuda externa. Aumentar o tamanho da coalizão vencedora nas nações em desenvolvimento oferece as melhores perspectivas de longo prazo para melhorar seu bem-estar econômico e social, porque a natureza da competição política em tais sistemas incentiva os líderes que desejam sobreviver no cargo a produzir políticas com esses objetivos em mente. A ajuda externa, muitas vezes impede esse objetivo. Os recursos gratuitos reduzem a dependência de um líder das 
receitas fiscais e, assim, removem a necessidade de os líderes aprovarem políticas que incentivem a atividade econômica. Na melhor das hipóteses a ajuda externa e a renda dos recursos naturais, induz um declínio na atividade econômica e no bem-estar social, além de retardar a democratização. Embora estes recursos forneçam aos líderes o necessário para promover o bem-estar social, na verdade oferecem o incentivo político para ter o comportamento oposto.

Da amostra de artigos relacionados com a renda do petróleo extraídos da base de dados da WoS, foi possível encontrar os principais autores em termos de quantidade de artigos encontrados na referida amostra, onde apenas quatro autores publicaram pelo menos quatro artigos. Destes autores, três possuem como publicações recentes sobre o tema no ano de 2020. As informações sobre os principais autores da referida amostra estão condensadas na Tabela 3 abaixo.

Tabela 3. Autores com maior número de publicações dos artigos da base de dados da WoS.

\begin{tabular}{|c|c|c|c|c|c|c|}
\hline Autor & $\begin{array}{l}\text { Qtde de } \\
\text { Arigos }\end{array}$ & $\begin{array}{l}\text { Qtde. } \\
\text { Citações }\end{array}$ & h-Index & Artigo Mais Recente & $\begin{array}{c}\text { Revista } \\
\text { Científica }\end{array}$ & $\begin{array}{c}\text { Ano de } \\
\text { Publicação }\end{array}$ \\
\hline Zaman, Khalid & 9 & 106 & 47 & $\begin{array}{l}\text { Determination of resource } \\
\text { curse hypothesis in } \\
\text { mediation of financial } \\
\text { development and clean } \\
\text { energy sources: Go-for- } \\
\text { green resource policies }\end{array}$ & $\begin{array}{l}\text { Resources } \\
\text { Policy }\end{array}$ & 2020 \\
\hline $\begin{array}{l}\text { Farzanegan, } \\
\text { Mohammad Reza }\end{array}$ & 6 & 80 & 22 & $\begin{array}{l}\text { Does oil rents dependency } \\
\text { reduce the quality of } \\
\text { education? }\end{array}$ & $\begin{array}{l}\text { Empirical } \\
\text { Economics }\end{array}$ & 2020 \\
\hline Auty, R.M. & 4 & 48 & 24 & $\begin{array}{l}\text { Natural resources and } \\
\text { 'gradual' reform in } \\
\text { Uzbekistan and } \\
\text { Turkmenistan }\end{array}$ & $\begin{array}{l}\text { Natural } \\
\text { Resources } \\
\text { Forum }\end{array}$ & 2003 \\
\hline $\begin{array}{l}\text { Nassani, } \\
\text { Abdelmohsen A. }\end{array}$ & 4 & 11 & 11 & $\begin{array}{l}\text { Determination of resource } \\
\text { curse hypothesis in } \\
\text { mediation of financial } \\
\text { development and clean } \\
\text { energy sources: Go-for- } \\
\text { green resource policies }\end{array}$ & $\begin{array}{l}\text { Resources } \\
\text { Policy }\end{array}$ & 2020 \\
\hline
\end{tabular}

Fonte: Autores.

Khalid Zaman foi identificado como o principal autor da amostra sobre os estudos relacionados com a renda do petróleo, com 9 publicações, no qual o referido autor possui 106 citações e um h-index de 47, um fator de impacto mais que o dobro do segundo principal autor em termos de artigos publicados. Os artigos publicados por este autor possuem como objeto de estudo a mensuração empírica do impacto sobre o meio ambiente das atividades ligadas ao petróleo e demais combustíveis fósseis, às questões ligadas as energias limpas e a gestão financeira do mercado de recursos naturais.

A sua publicação mais recente é o artigo Determination of resource curse hypothesis in mediation of financial development and clean energy sources: Go-for-green resource policies, publicado em 2020 na Resources Policy. Estudam a importância para a demanda de energia, dos recursos naturais e dos indicadores de desenvolvimento financeiro sobre as emissões de $\mathrm{CO} 2$, as emissões da combustão de combustíveis fósseis e as emissões de gases de efeito estufa no contexto da Arábia Saudita para o período 1975-2018, utilizando um modelo ARCH.

Segundo Anser et al. (2020), os resultados indicam que a demanda por energia elétrica aumenta as emissões de CO2 e as emissões de gases com efeito estufa, enquanto o valor adicionado da indústria eleva o consumo de combustíveis fósseis. 
Dentre outros resultados, foi confirmado a relação em U entre o PIB per capita e o consumo de combustíveis fósseis e a relação monotonicamente crescente entre o PIB per capita e emissão de CO2. Tais resultados tem implicações importantes para a questões de política econômica e de meio ambiente.

Mohammad Reza Farzanegan é o segundo principal autor da base de dados, no qual possui um h-index de 22, 80 citações e 6 artigos publicados sobre renda do petróleo. Seu artigo mais recente é o Does oil rents dependency reduce the quality of education? publicado em 2020 na Empirical Economics, fornecendo evidências empíricas com base nos efeitos negativos de longo prazo de aluguéis de petróleo na qualidade da educação. A análise contemplou mais de 70 países do período de 1995-2015. Identificou-se um efeito robusto e negativo de longo prazo (Farzanegan; Thum, 2020).

Outro autor identificado como um dos principais da amostra relacionados com a renda do petróleo, Richard M. Auty possui 4 publicações, 48 citações e um $h$-index de 24 . O artigo mais recente encontrado na referida amostra é Natural resources and 'gradual' reform in Uzbekistan and Turkmenistan, publicado em 2003 na Natural Resources Forum, no qual argumentou que o Uzbesquistão e o Turcomenistão, ricos em recursos naturais, consolidaram governos não desenvolvimentistas e aliviaram a pressão por reformas econômicas, de modo que os ativos básicos de geração de riqueza estavam sendo destruídos (Auty, 2003).

Abdelmohsen A. Nassani também é um dos principais autores da referida base de dados, com 4 publicações, 11 citações e um h-index de 11, no qual sua publicação mais recente é a mesma que foi identificada para Khalid Zaman (Anser et al., 2020), evidenciando uma existência de uma rede de colaboração quanto a produção científica.

Para a análise bibliométrica dos artigos relacionados com a renda do petróleo coletados na base de dados da WoS, a identificação das principais revistas científicas onde os artigos foram publicados podem revelar as áreas do conhecimento científico que mais contribuem para o desenvolvimento do tema. Em termos de quantidade de artigos publicados, a revista científica Resources Policy apresentou a maior quantidade de publicações, com 20 artigos, representando 10,99\% dos artigos relacionados com a renda do petróleo, além de possuir um fator de impacto, mensurado pelo fator JCR, de 3,986 para o ano de 2019, conforme apresentado pela Tabela 4 abaixo.

Tabela 4. Principais Revistas Científicas dos artigos relacionados com a renda do petróleo, extraídos da base de dados da WoS.

\begin{tabular}{lcccc}
\hline \multicolumn{1}{c}{ Revista Científica } & Artigos Publicados & \% dos artigos totais & ISSN & JCR (2019) \\
\hline Resources Policy & 20 & $10,99 \%$ & $0301-4207$ & 3,986 \\
Comparative Political Studies & 7 & $3,85 \%$ & $1552-3829$ & 3,197 \\
Journal of Internacional Development & 4 & $2,20 \%$ & $1099-1328$ & 1,225 \\
World Development & 4 & $2,20 \%$ & $1552-3829$ & 3,869 \\
Applied Economics & 3 & $1,65 \%$ & $1466-4283$ & 0,968 \\
Defence and Peac Economics & 3 & $1,65 \%$ & $1193-1207$ & 1,247 \\
Economic Change and Restructuring & 3 & $1,65 \%$ & $1573-9414$ & 1,500 \\
Economic Systems & 3 & $1,65 \%$ & $0939-3625$ & 1,578 \\
Ernegy Economics & 3 & $1,65 \%$ & $0140-9883$ & 5,203 \\
Enviromental Development and & 3 & $1,65 \%$ & $1573-2975$ & 2,191 \\
Sustainability & & & & \\
Enviromental Science and Pollution & 3 & $1,65 \%$ & $2455-0272$ & 3,056 \\
Research & 3 & $1,65 \%$ & $1531-5088$ & 5,000 \\
International Organization & 3 & $1,65 \%$ & $0022-3433$ & 2,419 \\
Journal of Peace Research & $\mathbf{6 2}$ & $\mathbf{3 4 , 0 7 \%}$ & $\mathbf{M e ́ d i a ~ J C R}$ & $\mathbf{2 , 7 2 6}$ \\
Total TOP 13 & 120 & $65,93 \%$ & & \\
Outras Revistas Científicas & $\mathbf{1 8 2}$ & $\mathbf{1 0 0 , 0 0 \%}$ & & \\
Total & & &
\end{tabular}


Para selecionar as principais revistas científicas, foi considerado como linha de corte as revistas que possuíam pelo menos três artigos da amostra publicados, onde as 13 principais revistas em conjunto aglutinam cerca de $34,07 \%$ das publicações que discutem a renda do petróleo e seus temas transversais. A média de fator de impacto das 13 revistas científicas apresentadas na Tabela 4 é de 2,726. A revista científica que possui o maior fator de impacto é a Ernegy Economics, com um fator JCR de 5,203 e a revista científica que possui o menor JCR $(0,968)$ é a Applied Economics.

Outro ponto a destacar sobre os periódicos científicos é que as principais áreas do conhecimento abarcadas pelos periódicos indicados são às ciências ambientais e áreas correlatas com o meio ambiente, ciências políticas e sociais e, principalmente, a ciência econômica. Estas áreas do conhecimento indicam o interesse das mesmas sobre o referido assunto, bem como no seu desenvolvimento, para o desenvolvimento científico e subsídio para eventuais implicações de políticas públicas.

\subsection{Análise de Redes de Cocitações e de Palavras-Chave}

Realizou-se neste estudo a análise da rede de cocitação dos autores utilizados como referências nos artigos da amostra, a partir da qual identificaram-se os principais autores. A relevância na constituição da rede de cocitação de autores se deve à identificação da base intelectual e analítica dos artigos relacionados com a renda do petróleo. Para a construção da referida rede, foram considerados os autores que obtiveram no mínimo 15 citações. São apresentados abaixo os 10 mais influentes e algumas das obras mais influentes por eles produzidas.

Dentre os autores identificados neste estudo, Michael L. Ross é o mais influente. Com 121 citações e um score de centralidade de 103,93, Ross é o principal autor sobre Resource Curse e seus impactos sobre diversos aspectos. Em sua publicação mais relevante "Does Oil Hinder Democracy?” concluiu de forma robusta que a disponibilidade de petróleo causa danos à democracia e seus impactos são ainda mais graves em economias mais pobres. Sendo o segundo autor mais influente, Jeffrey D. Sachs é um precursor nos estudos sobre a "Maldição dos Recursos Naturais". Juntamente com Andrew Warner desenvolveu uma série de estudos econométricos com o objetivo de testar empiricamente a existência de uma relação negativa entre dependência de recursos naturais e crescimento econômico. Com 109 citações e um score de centralidade de 98,82 é o segundo autor mais influente identificados neste estudo.

Paul Collier, com 104 citações e 88,93 de score de centralidade de rede de cocitações, desenvolveu importantes estudos relacionados ao desenvolvimento econômico e conflitos armados. Em seu estudo mais citado "Greed and grievance in civil war" desenvolveu um modelo econométrico sobre a iminência de uma guerra civil e concluiu que haveria maior probabilidade de incidência em países ricos em recursos naturais. Daron Acemoglu é uma referência nos estudos sobre a importância das instituições na performance econômica dos países. Dada a dificuldade em se identificar indicadores capazes de avaliar a qualidade das instituições, utilizou indicadores exógenos em seu estudo "The Colonial Origins of Comparative Development: An Empirical Investigation” para sugerir que há ganhos econômicos substanciais com o aprimoramento das instituições. Com 90 citações e um score de centralidade de 77,38, Acemoglu ocupa a quarta posição no ranking dos autores mais influentes.

Com 60 citações e um score de centralidade na rede de cocitação dos artigos relacionados com a renda de petróleo, Richard Auty, autor identificado também como um dos principais autores na Tabela 3 apresentada anteriormente, é também um autor importante para a constituição da base intelectual dos artigos selecionados na referida base de dados, cujo principal trabalho identificado é o livro Resource Abundance and Economic Development (Auty, 2001), no qual explora os fatores que influenciam o fraco desempenho dos países em desenvolvimento com abundância de recursos em comparação com os países com escassez de recursos. 
Frederick Van der Ploeg, com 59 citações e 52,32 de score de centralidade de rede de cocitações, apresenta como principal trabalho das citações o artigo Natural Resources: Curse or Blessing?, publicado no Journal of Economic Literature (Van der Ploeg, 2011), no qual o artigo analisa uma diversidade de hipóteses sobre os motivos pelos quais alguns países se beneficiam dos recursos naturais e outros perdem com a sua presença, no qual são discutidas as hipóteses de que uma posição favorável nos respectivos recursos induz a apreciação da taxa de câmbio real, desindustrialização, e baixa perspectiva de crescimento, no qual tais efeitos são mais profundos em países voláteis com instituições fracas e falta de Estado de direito, corrupção, democracias presidenciais, e sistemas financeiros subdesenvolvidos.

Thorvaldur Gylfason foi identificado como o sétimo principal autor na rede de citações, apresentando uma centralidade de 50,25 e com 58 citações. Seu principal trabalho relacionado com o tema é Natural resources, education, and economic development (Gylfason, 2001). O artigo apresenta que os recursos naturais trazem riscos e uma delas é que muitas pessoas ficam presas em indústrias baseadas em recursos naturais intensivos e de baixa qualificação, negligenciando a educação da geração atual e da geração vindoura. Os resultados sugerem que os recursos naturais inversamente relacionados com o capital humano, diminuindo assim o ritmo do desenvolvimento econômico.

Halvor Mehlum foi identificado como o oitavo principal autor na rede de citações, apresentando uma centralidade de 52,83 e com 54 citações. Seu principal trabalho relacionado com o tema é Institutions and the resource curse (Mehlum et al., 2006). Neste artigo os autores demonstram que a qualidade das instituições determina se esses países evitam ou não a maldição dos recursos. James A. Robinson foi identificado como o nono principal autor na rede de citações, apresentando uma centralidade de 40,65 com 41 citações, o principal trabalho do autor relacionado com o tema é Political Foundations Of The Resource Curse (Robinson; Torvick; Verdier, 2006). O artigo estudou que a geração de recursos e incentivos políticos são importantes para a eficiência, além de uma relação política nas eleições, podendo influenciar a má alocação de recursos no resto da economia. Países com instituições que promovem a prestação de contas e competência do estado, tendem a se beneficiar eficientemente de recursos, amenizando incentivos políticos perversos.

Christa N. Brunnschweiler foi identificada como o décimo principal autor na rede de citações, apresentando uma centralidade de 38,76 com 40 citações, o principal trabalho do autor é The resource curse revisited and revised: A Tale Of PAnd Red Herrings (Brunnschweiler; Bulte, 2008). O artigo estudou o uso de recursos para extração do petróleo e o impacto econômico. Concluiu-se que a dependência destes recursos não afeta o crescimento do país, impactando de forma positiva na economia. Cabe ressaltar que a rede de cocitação de autores relacionados com os estudos sobre a renda do petróleo está apresentada na Figura 1 a seguir. 
Figura 1. Rede de Cocitação de principais Autores sobre Renda do Petróleo da base de dados da WoS.

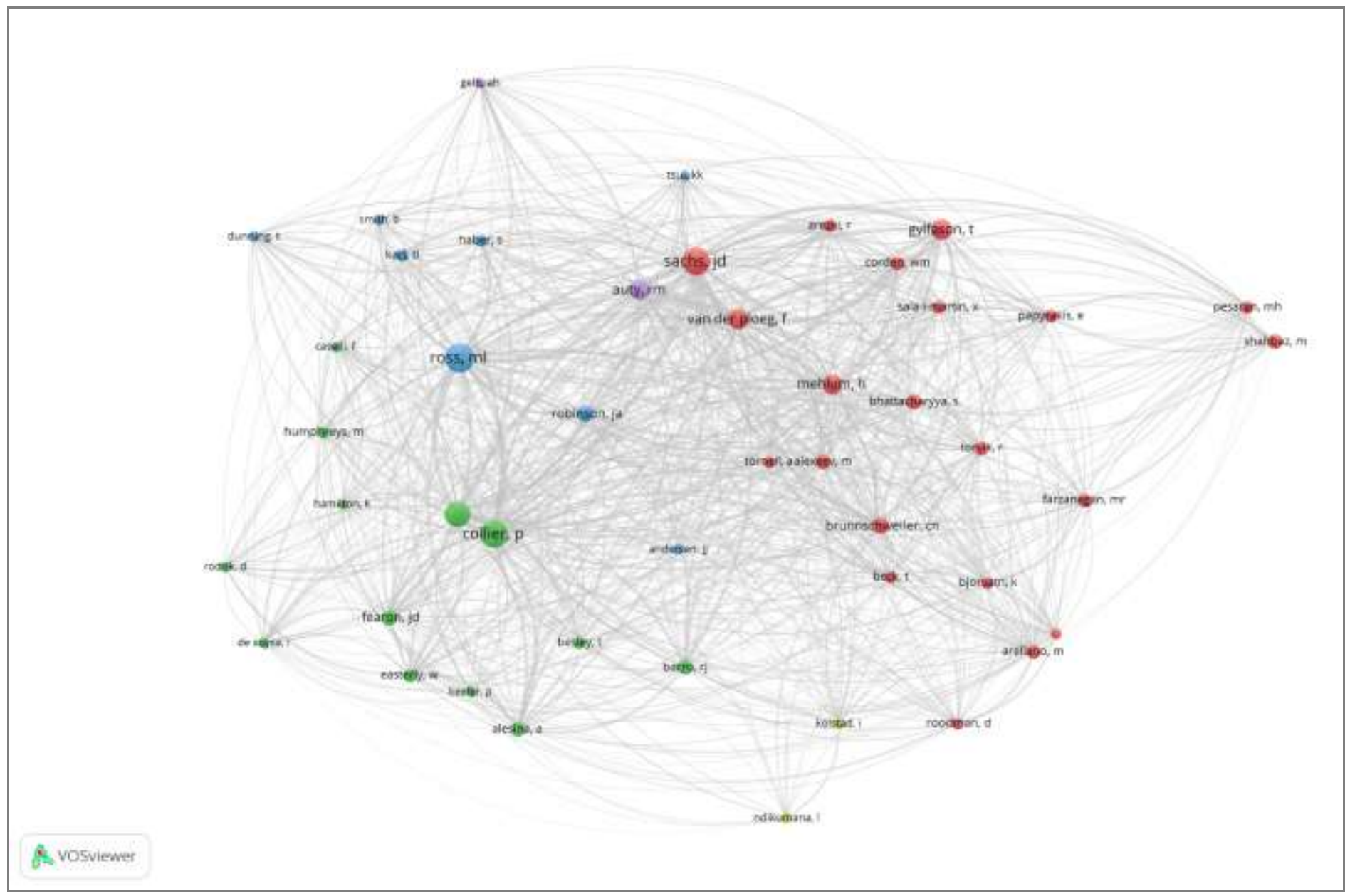

Fonte: Autores.

Quanto a rede de co-ocorrência de palavras-chave referente aos artigos relacionados com a renda do petróleo, as principais palavras-chave são "natural resources”, "growth”, “institutions”, “economic growth”, “oil”, “curse”, "democracy”, "oil rents", "resource curse” e "africa”, sendo estas palavras-chave em linha com as discussões realizadas nas análises bibliométricas realizadas até então. Algumas palavras-chave identificadas como principais chamam a atenção. A palavra-chave “curse” indica a preocupação dos benefícios ou os malefícios que a abundância de recursos naturais pode influenciar nas estratégias de desenvolvimento dos estados nacionais, denominado usualmente como maldição dos recursos naturais, traduzido pela palavra-chave "resource curse".

A expressão "democracy” demonstra uma preocupação de parte dos artigos na questão do impacto da abundância dos recursos naturais na condução política dos países, pelos quais regimes democráticos podem ser fragilizados pelos conflitos oriundos da repartição entre os entes da sociedade em relação ao excedente de renda oriundo dos recursos naturais. Tais conflitos, podem ser observados também em magnitude mundial, tendo em vista que o petróleo ainda continua sendo uma fonte importante de energia para o desenvolvimento da atividade produtiva, com efeitos de encadeamento e arrasto ainda relevantes no setor industrial.

A palavra-chave "africa” revela o interesse da discussão do tema da renda do petróleo e a abundância de recursos naturais no continente africano, tendo em vista que este continente possui reservas de petróleo e gás natural relevantes. Além disso, países como Nigéria e África do Sul figuram como principais países em termos de nacionalidade dos autores, conforme 
apresentado na Tabela 1, reforçando o argumento apresentado. As principais palavras-chave dos artigos da amostra são apresentadas na Figura 2.

Para identificar a tendencia das agendas de pesquisa sobre a discussão da renda do petróleo, a mesma Figura 2 apresenta as referidas palavras-chave em termos de incidência por ano e, neste sentido, com a incidência em períodos mais recentes, tal aspecto auxiliará na identificação das tendencias na referida agenda de pesquisa sobre o assunto. Foi identificado com relação à palavra-chave "economic growth", que além de ser uma das principais em termos de co-ocorrência, a sua incidência se dá em períodos recentes, revelando a preocupação com o impacto dos recursos naturais no crescimento econômico, reforçando a tese apresentada na primeira parte do artigo, sobre a existência da relação entre o aumento das publicações relacionadas com a renda do petróleo e a queda do preço do petróleo, revelando que esta temática tem sido uma preocupação proeminente para implicação de políticas públicas que resultem em estratégias de crescimento sustentado.

Além disso, a incidência recente de palavras-chave como "natural resource curse", "natural resource rents", "dependence", "abundance" e "countries" revelam a tendência de se discutir os efeitos da abundância e a dependência dos recursos naturais sobre o processo de desenvolvimento dos países, sobretudo associado à questão da discussão da maldição dos recursos naturais, que possui uma questão relevante para países que possuem tais recursos em abundância. $\mathrm{O}$ aprofundamento desta discussão tem vindo à tona em períodos recentes, conforme Figura 2 abaixo.

Figura 2. Rede de Co-ocorrência de Palavras-chave dos artigos coletados da base de dados do WoS de acordo com a incidência por ano de publicação.

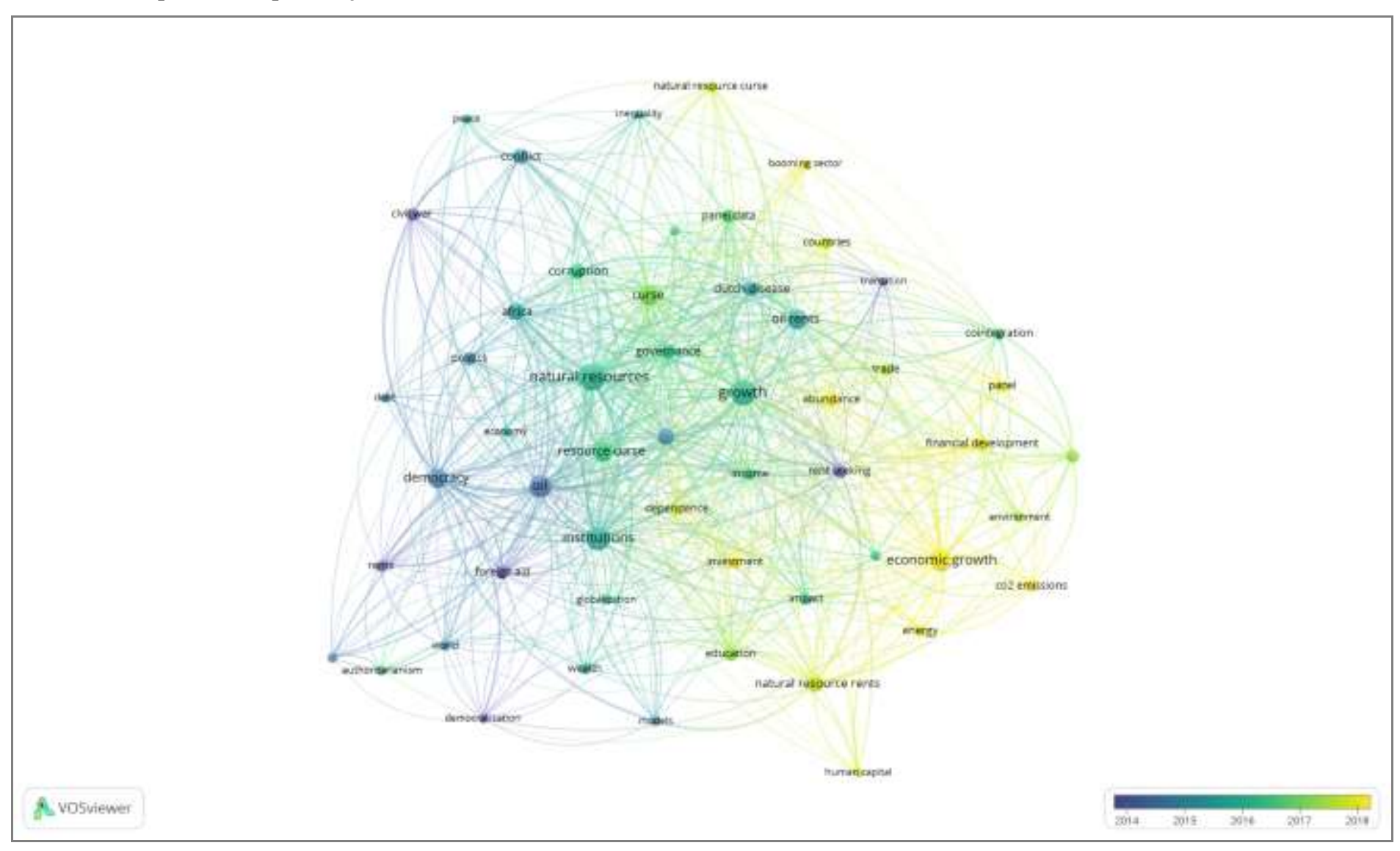

Fonte: Autores. 
Outra característica importante dos estudos mais recentes é a sua aplicação empírica, utilizando ferramentas matemáticas, estatísticas e econométricas, revelando a necessidade de teste das hipóteses discorridas sobre o tema de estudo, para verificar suas ocorrências nos países. Tal ponto pode ser verificado pelas palavras-chave "cointegration" e "panel".

Além disso, a palavra-chave "human capital" está relacionada com uma preocupação recente entre a renda do petróleo e o capital humano. O termo "financial development" se deve a atenção de artigos na estratégia de desenvolvimento baseado nos excedentes gerados pelos recursos nacionais para serem alocados em demais atividades econômicas de interesse da sociedade. Ademais, palavras-chave como "CO2 emissions", "energy" e "environment" revelam a importância da sustentabilidade e do cuidado com o meio ambiente, associado à produção de petróleo, que atualmente continua sendo uma matriz energética importante para a atividade econômica.

\section{Considerações Finais}

O objetivo do presente artigo é a realização de uma revisão da bibliografia relacionada com a renda do petróleo a partir da análise bibliométrica para organizar o conhecimento científico sobre o tema e as agendas de pesquisa. Adotando as palavras-chave descritas na metodologia, a amostra consiste em 182 artigos coletados na base científica Web of Science. A referida base indica o crescimento elevado de número de artigos publicados a partir de 2014, período coincidente com a queda do preço do petróleo, que impactou os países cujo eixo dinâmico da economia reside neste setor.

Quanto à nacionalidade dos autores relacionados com os artigos, foi identificado entre os principais países em termos de nacionalidade dos autores a Alemanha, Paquistão, China, Turquia, Canadá, Nigéria, Arábia Saudita e África do sul, que em conjunto com os Estados Unidos e a Inglaterra, concentram 58,24\% dos autores relacionados com os artigos sobre renda do petróleo, países estes que possuem uma produção relevante de petróleo e gás natural.

Dentre os principais artigos da amostra em termos de citação média por ano, o artigo Regional Favoritism foi identificado como o principal artigo. Khalid Zaman foi identificado como o principal autor da amostra sobre os estudos relacionados com a renda do petróleo, cujo artigo mais recente é Determination of resource curse hypothesis in mediation of financial development and clean energy sources: Go-for-green resource policies, publicado na Resources Policy.

$\mathrm{Na}$ análise das principais revistas cientificas, foi identificado que as principais áreas do conhecimento abarcadas pelos periódicos indicados são às ciências ambientais e áreas correlatas com o meio ambiente, ciências políticas e sociais e, principalmente, a ciência econômica. A revista científica identificada com maior fator de impacto foi Ernegy Economics e a revista que possui mais artigos publicados da amostra é European Resources Policy.

A rede de cocitação de autores apresentou os principais autores utilizados para conformação da base intelectual para os estudos relacionados com renda de petróleo, que em boa medida, discutem as implicações relacionadas com a abundância dos recursos naturais. Quanto a rede de co-ocorrência de palavras-chave, identificou-se as palavras-chave com maiores ocorrências nos artigos da amostra, que são "natural resources", "growth”, "institutions", “economic growth”, “oil”, “curse”, "democracy", "oil rents", "resource curse" e "africa".

Ademais, a rede de co-ocorrência de palavras-chave indica as palavras-chave que possuem incidência em períodos recentes, evidenciando a direção dos estudos para temas relacionados aos impactos sobre o crescimento econômico, a utilização de métodos empíricos para o estudo dos temas, discussão sobre a abundância, dependência e maldição dos recursos naturais, financiamento do desenvolvimento, relação entre capital humano e renda do petróleo, bem como temas ligados ao meio ambiente e sustentabilidade.

Conforme exposto no presente artigo, o foco da análise bibliométrica está relacionado com os estudos sobre renda do petróleo contidos na WoS, em uma perspectiva global. Assim, constitui-se como uma limitação do referido estudo a análise bibliométrica dos artigos publicados no Brasil, para realização de uma base comparada do estado-da-arte sobre os estudos 
relacionados com a renda do petróleo entre a perspectiva global e a perspectiva dos estudos no Brasil. Tal ponto pode ser um estudo futuro relevante para o conhecimento sobre o tema. Além disso, como proposta de estudos futuros, sugere-se intensificar as pesquisas sobre o impacto da renda do petróleo, envolvendo variáveis econômicas, políticas e sociais em diversos intervalos de tempo.

\section{Referências}

Acemoglu, D., Johnson, S., \& Robinson, J. A. (2001). The colonial origins of comparative development: An empirical investigation. American economic review, 91(5), 1369-1401.

Agência Nacional do Petróleo, Gás Natural e Biocombustíveis - ANP (2020). https://www.gov.br/anp/pt-br.

Andersen, J. J., \& Ross, M. L. (2014). The big oil change: A closer look at the Haber-Menaldo analysis. Comparative Political Studies, 47(7), 993-1021.

Anser, M. K., Yousaf, Z., Zaman, K., Nassani, A. A., Alotaibi, S. M., Jambari, H., \& Kabbani, A. (2020). Determination of resource curse hypothesis in mediation of financial development and clean energy sources: Go-for-green resource policies. Resources Policy, 66, 101640.

Auty, R. (2003). Natural resources and 'gradual'reform in Uzbekistan and Turkmenistan. In Natural Resources Forum, 27, $255-266$.

Auty, R. M. (Ed.). (2001). Resource abundance and economic development. Oxford University Press.

Aytaç, S. E., Mousseau, M., \& Örsün, Ö. F. (2016). Why some countries are immune from the resource curse: The role of economic norms. Democratization, 23(1), 71-92.

Barbosa, J. S. K., \& Reinert, M. (2014). Open Innovation: uma análise bibliométrica do período de 2003 a 2013. Anais do Encontro Nacional da Associação Nacional de Pós-Graduação e Pesquisa em Administração, 38.

BP (2020). Statistical Review of World Energy. https://www.bp.com/en/global/corporate/energy-economics/statistical-review-of-world-energy.html.

Brunnschweiler, C. N., \& Bulte, E. H. (2008). The resource curse revisited and revised: A tale of paradoxes and red herrings. Journal of environmental economics and management, 55(3), 248-264.

Chain, C. P., Santos, A. C. D., Castro, L. G. D., \& Prado, J. W. D. (2019). Bibliometric analysis of the quantitative methods applied to the measurement of industrial clusters. Journal of Economic Surveys, 33(1), 60-84.

Collier, P., \& Hoeffler, A. (2009). Testing the neocon agenda: Democracy in resource-rich societies. European Economic Review, 53(3), 293-308.

Cruz, B. D. O., \& Ribeiro, M. B. (2009). Sobre Maldições e Bênçãos: é possível gerir recursos naturais de forma sustentável? Uma análise sobre os royalties e as compensações financeiras no Brasil.

De Mesquita, B. B., \& Smith, A. (2008). Retesting selectorate theory: Separating the effects of W from other elements of democracy. American Political Science Review, 393-400.

De Mesquita, B. B., \& Smith, A. (2010). Leader survival, revolutions, and the nature of government finance. American journal of political science, 54(4), 936950.

Djankov, S., Montalvo, J. G., \& Reynal-Querol, M. (2008). The curse of aid. Journal of economic growth, 13(3), 169-194.

do Prado, J. W., de Castro Alcântara, V., de Melo Carvalho, F., Vieira, K. C., Machado, L. K. C., \& Tonelli, D. F. (2016). Multivariate analysis of credit risk and bankruptcy research data: a bibliometric study involving different knowledge fields (1968-2014). Scientometrics, 106(3), 1007-1029.

Farzanegan, M. R., \& Thum, M. (2020). Does oil rents dependency reduce the quality of education? Empirical Economics, 58(4), $1863-1911$.

Gylfason, T. (2001). Natural resources, education, and economic development. European economic review, 45(4-6), 847-859.

Hartwick, J. M. (1977). Intergenerational equity and the investing of rents from exhaustible resources. The american economic review, 67(5), 972-974.

Hassan, S. U., Haddawy, P., \& Zhu, J. (2014). A bibliometric study of the world's research activity in sustainable development and its sub-areas using scientific literature. Scientometrics, $99(2), 549-579$.

Hodler, R., \& Raschky, P. A. (2014). Regional favoritism. The Quarterly Journal of Economics, 129(2), 995-1033.

Hotelling, H. (1931). The economics of exhaustible resources. Journal of political Economy, 39(2), 137-175.

Humphreys, M., Sachs, J. D., Stiglitz, J. E., Soros, G., \& Humphreys, M. (2007). Escaping the resource curse. Columbia University Press.

Instituto Brasileiro de Petróleo - IBP (2020). Observatório do Setor de Petróleo e Gás. https://www.ibp.org.br/observatorio-do-setor/.

International Energy Agency - IEA (2020). World Energy Outlook 2020. https://www.iea.org/reports/world-energy-outlook-2020.

Khan, M. M., Zaman, K., Irfan, D., Awan, U., Ali, G., Kyophilavong, P., \& Naseem, I. (2016). Triangular relationship among energy consumption, air pollution and water resources in Pakistan. Journal of cleaner production, 112, 1375-1385. 
Research, Society and Development, v. 10, n. 9, e38110918320, 2021

(CC BY 4.0) | ISSN 2525-3409 | DOI: http://dx.doi.org/10.33448/rsd-v10i9.18320

Mehlum, H., Moene, K., \& Torvik, R. (2006). Institutions and the resource curse. The economic journal, 116(508), 1-20.

Okada, K., \& Samreth, S. (2017). Corruption and natural resource rents: evidence from quantile regression. Applied Economics Letters, 24(20), 1490-1493.

Ozturk, I. (2017). Measuring the impact of alternative and nuclear energy consumption, carbon dioxide emissions and oil rents on specific growth factors in the panel of Latin American countries. Progress in Nuclear Energy, 100, 71-81.

Pamplona, J. B., \& Cacciamali, M. C. (2017). O paradoxo da abundância: recursos naturais e desenvolvimento na América Latina. Estudos Avançados, 31(89), 251-270.

Postali, F. A. S., \& Nishijima, M. (2011). Distribuição das rendas do petróleo e indicadores de desenvolvimento municipal no Brasil nos anos $2000 \mathrm{~S}$. Estudos Econômicos (São Paulo), 41(2), 463-485.

Ramos-Rodríguez, A. R., \& Ruíz-Navarro, J. (2004). Changes in the intellectual structure of strategic management research: A bibliometric study of the Strategic Management Journal, 1980-2000. Strategic management journal, 25(10), 981-1004.

REIS, C. D. B. (2012). Recursos naturais e desenvolvimento econômico: da especialização à diversificação produtiva e exportadora nos Seanic's. $232 f$ (Doctoral dissertation, Tese (Doutorado)-Universidade Federal do Rio de Janeiro).

Robinson, J. A., Torvik, R., \& Verdier, T. (2006). Political foundations of the resource curse. Journal of development Economics, 79(2), 447-468.

Sachs, J. D. (2007). How to handle the macroeconomics of oil wealth. Escaping the resource curse, 180.

Shahbaz, M., Destek, M. A., Okumus, I., \& Sinha, A. (2019). An empirical note on comparison between resource abundance and resource dependence in resource abundant countries. Resources Policy, 60, 47-55.

Thies, C. G. (2010). Of rulers, rebels, and revenue: State capacity, civil war onset, and primary commodities. Journal of peace research, 47(3), 321-332.

Udi, J., Bekun, F. V., \& Adedoyin, F. F. (2020). Modeling the nexus between coal consumption, FDI inflow and economic expansion: does industrialization matter in South Africa? Environmental Science and Pollution Research, 1-12.

Van der Ploeg, F. (2011). Natural resources: curse or blessing? Journal of Economic literature, 49(2), 366-420.

Van Eck, N. J., \& Waltman, L. (2010). Software survey: VOSviewer, a computer program for bibliometric mapping. scientometrics, 84(2), 523-538.

Van Eck, N. J., \& Waltman, L. (2014). Visualizing bibliometric networks. In Measuring scholarly impact (pp. 285-320). Springer, Cham.

Van Eck, N. J., Waltman, L., Dekker, R., \& van den Berg, J. (2010). A comparison of two techniques for bibliometric mapping: Multidimensional scaling and VOS. Journal of the American Society for Information Science and Technology, 61(12), 2405-2416. 